\title{
Endometriosis Misdiagnosed as a Metastatic Ovarian Tumour
}

\author{
J. O. Imaralu1 ${ }^{*}$, M. A. Ajani², M. A. Adesina ${ }^{3}$, N. K. Ojo ${ }^{4}$ \\ ${ }^{1}$ Department of Obstetrics and Gynaecology, Babcock University Teaching Hospital, Ilishan-Remo, Nigeria \\ ${ }^{2}$ Department of Pathology, Babcock University Teaching Hospital, Ilishan-Remo, Nigeria \\ ${ }^{3}$ Department of Surgery, Babcock University Teaching Hospital, Ilishan-Remo, Nigeria \\ ${ }^{4}$ Department of Radiology, Babcock University Teaching Hospital, Ilishan-Remo, Nigeria \\ Email: *imaraluj@babcock.edu.ng
}

How to cite this paper: Imaralu, J.O., Ajani, M.A., Adesina, M.A. and Ojo, N.K. (2019) Endometriosis Misdiagnosed as a Metastatic Ovarian Tumour. Case Reports in Clinical Medicine, 8, 189-195. https://doi.org/10.4236/crcm.2019.87023

Received: May 19, 2019

Accepted: July 9, 2019

Published: July 12, 2019

Copyright ( 2019 by author(s) and Scientific Research Publishing Inc. This work is licensed under the Creative Commons Attribution International License (CC BY 4.0).

http://creativecommons.org/licenses/by/4.0/ (c) () Open Access

\begin{abstract}
Endometriosis is a debilitating problem with pain in the short term and high risk of infertility later. It is an oestrogen-dependent condition found in about $10 \%$ of women of reproductive age, about $1 / 3$ of infertile women and as high as $80 \%$ of women with chronic pelvic pain. The condition is not well understood and thus associated with misdiagnosis and delayed diagnosis. Higher rates of misdiagnosis occur in blacks and this is especially for pelvic tumors-fibroids and ovarian tumors. We present here the case of a 30-year-old nullipara, who had an umbilical nodule (Sister Mary Joseph's) and was found on imaging to have a pelvic tumor which was suspected to be an ovarian cancer. Diagnostic laparoscopy during the menstrual phase however revealed endometriosis in early stage. Misdiagnosis of endometriosis has potential to distort the course of the disease and endanger fertility prospects; early laparoscopic evaluation of patients with unclear pelvic pathologies would help to prevent this occurrence.
\end{abstract}

\section{Keywords}

Diagnosis, Endometriosis, Pelvis, Tumour, Treatment, Imaging

\section{Introduction}

Endometriosis is often symptomatic when present, causing debilitating illness in many cases. It is the occurrence of endometrium-like glands and stroma outside the uterine cavity [1].

Endometriosis affects about $10 \%$ of women of reproductive age 15 - 49 years. 
It has been implicated as one of the top three causes of female infertility [2].

Endometriosis can also be described as a disease of theories as the cause and course has been explained by findings that suggest various theories [1] [2] [3]. Endometriosis was thought to be rare among black populations; evolving knowledge however continued to unravel this myth. Misdiagnosed endometriosis is a recognized factor in treatment delays and worsening conditions, leading to infertility and chronic pelvic pain, especially in blacks [2]. Endometriomas have been misdiagnosed for pelvic tumors mainly; fibroids and malignant ovarian tumors [2]. Endometrioma being misdiagnosed for a metastatic tumor is a rare occurrence.

We present here the case of a 30-year-old nullipara, who had a peri-umbilical nodule (Sister Mary Joseph's) and was found on imaging to have a pelvic tumor which was suspected to be a metastatic ovarian cancer. Laparoscopy during the menstrual phase, together with tissue histology however revealed endometriosis with tubal masses.

\section{Case Report}

A 30-year-old single nullipara, presented to the gynae clinic with one year history of lower abdominal pain which had become worse and continuous in the 2 months preceding her presentation to the hospital. She had significant weight loss but no history of cough, haemoptysis, abnormal uterine bleeding, or bleeding from other body orifices. She had used a non-steroidal anti-inflammatory drug (Diclofenac tablets) for 1 year and at the time of presentation; she had an upper gastrointestinal tract endoscopy done which showed that she had developed gastritis.

Examination revealed a young woman, with significant weight loss, but no pallor or lymph node enlargement. There was a nodular firm, non-tender $2 \mathrm{~cm}$ diameter spherical growth occupying the right half of the umbilicus. There was mild suprapubic tenderness and ascites was demonstrable by shifting dullness. She had a pinhole cervical os and a boggy, soft, tender Pouch of Douglas (POD), and a tender firm mass within it (Figure $1 \&$ Figure 2).

There was no adnexal or cervical motion tenderness. An assessment of a metastatic ovarian tumor was made. Ultrasound scanning (USS) and Computerized tomography $(\mathrm{CT})$ revealed a huge ovarian mass completely obliterating the POD with suspected metastasis to the anterior abdominal wall (Figure 3).

The CA-125 level was $<3.5 \mathrm{MIU} / \mathrm{ml}$, while her CEA was $<3.5 \mathrm{MIU} / \mathrm{ml}$ both were normal for laboratory reference range. Serum quantitative $\beta$-HCG assay was $<50 \mathrm{miu} / \mathrm{ml}$.

She was scheduled for laparoscopy, but shortly before the procedure she started menstruating with associated severe dysmenorrhea. Before laparoscopy, she had pelvic examination under anaesthesia, which revealed a cervical external os diameter $\approx 3 \mathrm{~mm}$, with scanty bleeding from the cervical os. Operative findings included haemoperitoneum with a covering layer of clearer peritoneal fluid, which upon suctioning revealed a bulky uterus with two huge tubal masses 


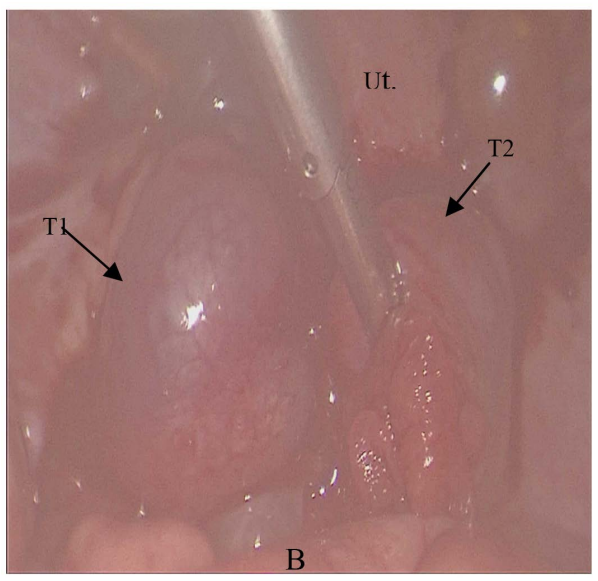

Figure 1. Tubal masses (T) in the posterior cul de sac mimicking a malignant ovarian tumor (Ut. = uterus, $\mathrm{B}=$ bowel).

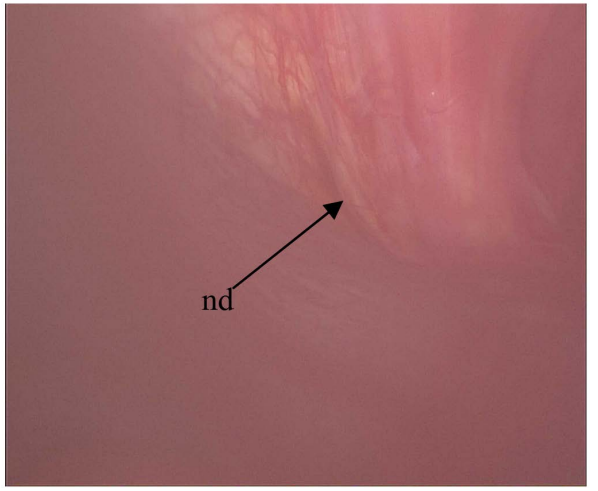

Figure 2. Laparoscopic view of the peritoneal surface of the umbilical nodule (nd).

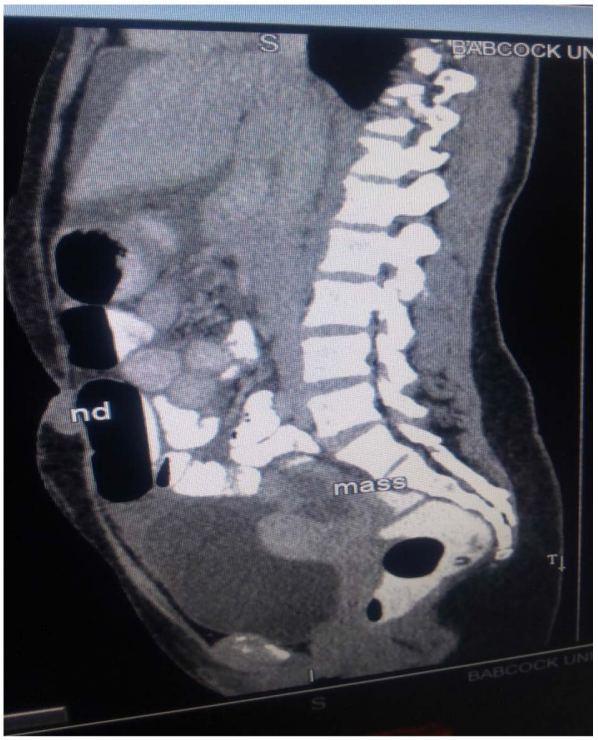

Figure 3. CT scan view showing the Mass in the POD and the umbilical nodule (nd). 
(mid-tubal diameter $\approx 6 \mathrm{~cm}$ each), posterior to the uterus and filling up the POD, there was continuous efflux of fresh blood from the dilated fimbrial ostia. The ovaries were covered by the tubal masses, they looked grossly normal, but had transparent haemorrhagic cysts on their surfaces. The peritoneal surface of the umbilicus had a well circumscribed $2 \mathrm{~cm}$ diameter nodular growth; which together with the cysts on the ovaries were excised for histology. Peritoneal fluid was also obtained for cytology and mycobacterial assay. The cervical external os was then dilated, to allow outflow of menstrum. The ascitic fluid cytology revealed reactive mesothelial cells with no evidence of atypical or malignant cells seen. It was also negative for acid fast bacilli.

Histological sections of the ovarian cystic mass and umbilical nodules showed islands of endometrial glands and endometrial stroma (Figure 4).

The glands contained a single layer of tall columnar cells and there were no foci of atypia or malignancy, features adjudged as consistent with endometriosis. She was commenced on dydrogestrone and her dysmenorrhea and pelvic pain had significantly reduced. Repeat ultrasonography done one month and repeated two months later confirmed absence of any pelvic tumors.

\section{Discussion}

Continuous epigastric pain made this patient first present to the gastroenterologist where endoscopic findings confirmed a probable sequela of analgesic abuse. The benefit of long term use of non-selective NSAIDs, for treatment of endometriosis, is limited by their adverse effects on the gastrointestinal tract [4]. This finding may also explain her weight loss from anorexia as patients with gastric ulcers typically have pain exacerbations after meals [5].

The huge tubal masses observed at laparoscopy, the demonstration of retrograde menstruation and the histologic confirmation of peritoneal endometriotic



Figure 4. Photomicrograph of the ovarian cystic mass showing islands of endometrial glands EG (arrow) and endometrial stroma ES (asterisk) consistent with endometriosis of the ovary. (Haematoxylin and eosin stains, $\times 400$.) 
deposits largely clarify the diagnosis of endometriosis. Laparoscopy done in the menstrual phase of the cycle afforded us this opportunity. There is currently no evidence in support of menstruation being an absolute contraindication to elective laparoscopy. It has however been recommended that laparoscopic resection of peritoneal endometriosis, should be avoided during the luteal phase because of the high recurrence rate observed when compared to other phases of the menstrual cycle [6].

The contiguous location of the ovary to the uterine tubes in the pelvis makes recognition of these organs in disease-states difficult. The cul de sac uterine tubal masses in this patient were misdiagnosed on ultrasound scanning and CT for a malignant ovarian tumor. Normal ovarian tissue with minor thin walled cysts on their surfaces which were excised and confirmed with histology suggested early stage endometriomas. Ovarian endometriomas have been demonstrated in histopathological specimens to start initially as extra-ovarian cysts with ectopic endometrium lining the surface of the cortex, which later gets invaginated to form an intra-ovarian pseudocyst [3].

The nodular umbilical growth in this patient was painless and had never bled, although it had increased in size over 12 months. This finding raised suspicion of malignancy as it has been reported in literature that $88 \%$ of umbilical malignancies originate outside the umbilicus [7]. The histologic finding of benign fibrocollagenous tissues however put our suspicions to rest and supports earlier reports that $60 \%$ of umbilical nodules are benign [8]. Ovarian and endometrial cancer reportedly account for $28 \%$ of Sister Mary Joseph's nodules [9]. Endometriosis has not been clearly shown in systematic reviews to increase the risk of ovarian cancer. However when ovarian cancer develops, the clear cell and endometrioid histologic types appear more likely [10] [11].

Intraoperative cervical dilatation significantly reduced the size of the tubal masses before the end of laparoscopy. Ultrasound scanning done during subsequent menstruations confirmed that the pelvic masses were no longer present. It had been reported that significantly more retrograde menstruation occurred with cervical external os diameter $<4.5 \mathrm{~mm}$ [12].

\section{Conclusion}

Misdiagnosis of endometriosis has potential to distort the course of the disease and endanger fertility prospects; early laparoscopic evaluation of patients with unclear pelvic pathologies would help to prevent this occurrence.

\section{Ethical Consideration}

The patient gave a written informed consent permitting the authors to publish this case report, while ensuring that her identity is not mentioned.

\section{Authors' Contributions}

IJO performed the initial clinical assessment and the laparoscopy together with 
AMA. AMA performed the histopathology and prepared the histopathological report, while ONK coordinated the radio-diagnosis. The initial draft of the manuscript was written by IJO with assistance from AMA. All authors read and approved the manuscript for publication.

\section{Acknowledgements}

Authors express sincere gratitude to the patient for permission to publish the case report. We also thank the consultant radiologists of Babcock University and Prisms Diagnostic Centre.

\section{Conflicts of Interest}

The authors declare that there is no competing interest relating to this case report.

\section{References}

[1] Adamson, G.D., Kennedy, S. and Hummeshoj, L. (2010) Creating Solutions in Endometriosis: Global Collaboration through the World Endometriosis Research Foundation. Journal of Endometriosis and Pelvic Pain Disorders, 2, 3-6. https://doi.org/10.1177/228402651000200102

[2] Bulletti, C., Coccia, M.E., Battistoni, S. and Borini, A. (2010) Endometriosis and Infertility. Journal of Assisted Reproduction and Genetics, 27, 441-447. https://doi.org/10.1007/s10815-010-9436-1

[3] Brosens, I., Puttemans, P., Gordts, S.Y., Campo, R., Gordts, S. and Benagiano, G. (2013) Early Stage Management of Ovarian Endometrioma to Prevent Infertility. Facts, Views \& Visions in ObGyn, 5, 309-314.

[4] Zhang, W.Y., Li, W. and Po, A. (1998) Efficacy of Minor Analgesics in Primary Dysmenorrhoea: A Systematic Review. BJOG: An International Journal of Obstetrics \& Gynaecology, 105, 780-789. https://doi.org/10.1111/j.1471-0528.1998.tb10210.x

[5] Spiller, R.C. (2001) Anorexia, Nausea, Vomiting and Pain. British Medical Journal, 323, 1354-1357. https://doi.org/10.1136/bmj.323.7325.1354

[6] Schweppe, K.W. and Ring, D. (2002) Peritoneal Defects and the Development of Endometriosis in Relation to the Timing of Endoscopic Surgery during the Menstrual Cycle. Fertility and Sterility, 78, 763-766. https://doi.org/10.1016/S0015-0282(02)03431-3

[7] Papalas, J.A. and Selim, M.A. (2011) Metastatic vs Primary Malignant Neoplasms Affecting the Umbilicus: Clinicopathologic Features of 77 Tumors. Annals of Diagnostic Pathology, 15, 237-242. https://doi.org/10.1016/j.anndiagpath.2010.12.004

[8] Galvan, G. (1999) Sister May Joseph's Nodule. Its Clinical Significance and Management. Anales de Medicina Interna, 16, 365-370.

[9] Fratellone, P.M. and Holowecki, M.A. (2009) Forgotten Node: A Case Report. World Journal of Gastroenterology, 15, 4974-4975. https://doi.org/10.3748/wjg.15.4974

[10] Zafrakas, M., Grimbizis, G., Timologou, A. and Tarlatzis, B. (2014) Endometriosis and Ovarian Cancer Risk: A Systematic Review of Epidemiological Studies. Frontiers in Surgery, 1, 1-6. 
[11] Sayasneh, A., Tsivos, D. and Crawford, R. (2011) Endometriosis and Ovarian Cancer: A Systematic Review. ISRN Obstetrics and Gynecology, 2011, Article ID: 140310. https://doi.org/10.5402/2011/140310

[12] Barbieri, R.L., Callery, M. and Perez, S.E. (1992) Directionality of Menstrual Flow: Cervical Os Diameter as a Determinant of Retrograde Menstruation. Fertility and Sterility, 57, 727-730. https://doi.org/10.1016/S0015-0282(16)54950-4 